\title{
Bilateral Breast Metastasis of Atypical Pulmonal Carcinoid
}

\author{
Pavlišta D* \\ Department of Oncogynecology, Charles University, Czech Republic
}

*Corresponding author: Pavlišta D, Department of Oncogynecology, Clinic of Obstetrics and Gynecology of the 1st Faculty of Medicine of Charles University and General Hospital in Prague, Czech Republic.

To Cite This Article: Pavlišta D, Bilateral Breast Metastasis of Atypical Pulmonal Carcinoid. 2020 - 7(6). AJBSR.MS.ID.001213.

DOI: 10.34297/AJBSR.2020.07.001213.

Received: 濫 February 28, 2020; Published: March 05, 2020

\begin{abstract}
The breast is an uncommon site of metastasis. We report a case of bilateral metastasis of atypical pulmonal carcinoid in the breast. Review of the literature has demonstrated only a few cases of carcinoid metastatis in a single breast, but not bilateral. In spite of the rarity of this finding, the differential diagnosis between primary and metastatic carcinoid or malignant tumor of the breast at all, should be considered in view of the different management of these two settings. Immunohistology has proved a very helpful method in this field. Accurate diagnosis of breast metastasis is important to avoid unnecessary treatment.
\end{abstract}

\section{Case report}

From the patient's history: her mother died of breast cancer and she had a breast surgery for a fibroadenoma in 1993. In January 2001 she had undergone an upper lobectomy of the right lung for an atypical bronchial carcinoid ( pT1, N2, M0, G2). An adjuvant radiotherapy was performed for lymph node metastasis. A follow-up after one year revealed a small metastatic tumor in the epigastrium. Additional metastasis were not found. She received six cycles of chemotherapy containing Vepeside and Cisplatin.

Our patient, a 42-year-old woman, presented to the tumor bord for surgical treatment of bilateral lesions in the breast. The results of the mammogram and physical examination revealed $1 \times 1$ and $2 \times 1 \mathrm{~cm}$ masses, bilateral in the upper outer quadrant of the breast and was thought to probably be benign. The results of the sonography confirmed these findings. There was no sign of axillary lymphadenopathy. The patient refused a core-cut biopsy.

The patient underwent bilateral lumpectomy. The result from the frozen section was not evident, so surgery was not extended. The final histological examination revealed two masses with ductal carcinoma, NOS (non-other specify), with lymphangiosis carcinomatosa, grading 3. Additionally, immunohistochemical analysis of all tumors was performed. The immunological features of lungcells, nodi metastasis and breast tumor were compared. This demonstrated positive results in all parameters typical for carcinoid tumor (chromogranin, NSE, Bombesin, Calcitonin, HISL 19) and negative results for ACTH and hCG. This description was compatible with multifocal metastasis of a carcinoid tumor.

\section{Comment}

Carcinoid tumors are slow-growing neoplasms derived from the endocrine cells of the gastrointestinal tract, bronchus and elsewhere. The carcinoid syndrome is classically due to an ileal carcinoid tumor with hepatic metastases. Carcinoid cells make up the neuro-endocrine cell system of the body (APUD system) and can produce various polypeptides, including serotonin. When the primary tumor is extraintestinal, the carcinoid syndrome may be produced without hepatic metastases. The first reported case of carcinoid tumor metastatic to the breast appeared in the literature in 1957 and only a small number of cases have been reported since then [1].

The existence of primary carcinoid tumors of the breast is still a controversial area in pathology. Several authors believe in the existence of primary carcinoid tumors of the breast [2]. Breast metastases from non-mammary malignant neoplasms are 
approximately $1-2 \%$ of breast tumors [3]. However, careful postmortem examination of the breast in cases of neoplasm would probably reveal more cases. The most common primary tumors that metastasis to the breast are contralateral breast carcinoma, melanoma, lymphoma, and leukemia [4].

This case demonstrates the difficulty in making the right diagnosis. The major problem for establishing an accurate diagnosis is to differentiate primary and metastatic mammary neoplasms. Failure to make a correct preoperative or intraoperative (frozen section) diagnosis of the tumor is common [5]. Histological differences of tumors in this case could have called for chemotherapy. The correct diagnosis was found through immunohistochemical analysis. In our opinion, when a carcinoid tumor or metastases of a malignant tumor are suspected, the diagnosis should be confirmed by immuno - histochemical analysis preoperative. If the diagnosis of metastatic tumor is confirmed, a limited operation, without mastectomy and axillary lymph node dissection, may be considered. This approach to the surgical treatment brings benefits to the patient, mainly before oncological treatment.

\section{References}

1. Kashlan R, Powell RW, Nolting SF (1982) Carcinoid and other tumors metastatic to the breast. J Surg Oncol 20(1): 25-30.

2. Hartgrink HH, Lagaay MB, Spaander PJ, Mulder H, Breslau PJ (1995) A series of carcinoid tumours of the breast. Eur J Surg Oncol 21(6): 609612.

3. Amichetti M, Perani B, Boi S (1990) Metastases to the breast from extramammary malignancies. Oncology 47(3): 257-260.

4. Moir GC, Carpenter R, Bass P, Royle GT (1993) Metastatic carcinoid of the breast: an unusual screen-detected breast cancer. Eur J Surg Oncol 19(1): 92-94.

5. Rubio IT, Korourian S, Brown H, Cowan C, Klimberg VS (1998) Carcinoid tumor metastatic to the breast. Arch Surg 133(10): 1117-1119. 